\title{
Computer-Generated Three-Dimensional Airway Models as a Decision-Support Tool for Preoperative Evaluation and Procedure-Planning in Pediatric Anesthesiology
}

\author{
Benjamin Kloesel ${ }^{1}$ (1) $\cdot$ Bethany Juhnke $e^{2,3} \cdot$ Laura Irvine $^{3} \cdot$ James V. Donadio IV ${ }^{3} \cdot$ Arthur Erdman $^{2,3} \cdot$ Kumar Belani $^{1}$
}

Received: 1 September 2020 / Accepted: 9 December 2020 / Published online: 11 January 2021

(C) The Author(s), under exclusive licence to Springer Science+Business Media, LLC part of Springer Nature 2021

\begin{abstract}
Technology improvements have rapidly advanced medicine over the last few decades. New approaches are constantly being developed and utilized. Anesthesiology strongly relies on technology for resuscitation, life-support, monitoring, safety, clinical care, and education. This manuscript describes a reverse engineering process to confirm the fit of a medical device in a pediatric patient. The method uses virtual reality and three-dimensional printing technologies to evaluate the feasibility of a complex procedure requiring one-lung isolation and one-lung ventilation. Based on the results of the device fit analysis, the anesthesiology team confidently proceeded with the operation. The approach used and described serves as an example of the advantages available when coupling new technologies to visualize patient anatomy during the procedural planning process.
\end{abstract}

Keywords Airway management $\cdot$ Pediatric anesthesiology $\cdot$ Three-dimensional airway model $\cdot$ Medical device $\cdot$ Device fit analysis

\section{Introduction}

In recent years, medical advancements in diagnosis and treatment of diseases have been closely associated with progress in technology. Imaging capabilities have benefitted from improved computing power and the development of systems with faster scanning times and higher resolution. While in the past, imaging modalities were primarily used for the evaluation of a patient's anatomy and the diagnosis of diseases,

Benjamin Kloesel and Bethany Juhnke contributed an equal amount of work and are both co-first authors

This article is part of the Topical Collection on Patient Facing Systems

Benjamin Kloesel

bkloesel@umn.edu

1 Department of Anesthesiology, Division of Pediatric

Anesthesiology, University of Minnesota, B515 Mayo Building, 420

Delaware Street SE, Minneapolis, MN 55455, USA

2 Earl E. Bakken Medical Devices Center, University of Minnesota, Minneapolis, MN, USA

3 Department of Mechanical Engineering, University of Minnesota, Minneapolis, MN, USA nowadays they can assist in planning of interventions such as surgery and radiation.

\section{Case study}

We present a case of a pediatric patient diagnosed with Niemann Pick disease type B and pulmonary (lung) alveolar proteinosis. For the latter diagnosis, the patient needed to undergo sequential whole lung lavage to improve pulmonary function in preparation for possible hematopoietic stem cell transplantation to treat her Nieman Pick disease. In pulmonary alveolar proteinosis, lung surfactants (lipoprotein complexes that reduce surface tension and help to keep the lungs expanded) accumulate in the alveolar space, thereby reducing the available area for gas exchange resulting in shortness of breath, low blood oxygen levels and later respiratory failure [1].

General anesthesia is required for sequential whole lung lavage. The airway is usually secured with a double-lumen endotracheal tube to allow lung isolation (separation of the airways from the left and right lung). While one lung is continuously being ventilated, the other lung is subjected to repeated instillation of saline solution followed by evacuation of 
the instilled fluid [2]. The therapeutic goal of this procedure is to wash out proteinaceous material in the lungs, which impairs gas exchange. Lung isolation is critical as inadequate separation of the lungs would lead to spillover of saline solution. Presence of large amounts of saline solution in both lungs would present as drowning and lead to significant morbidity and potentially death of the patient.

In adult patients, several lung isolation methods such as double-lumen endotracheal tubes and bronchial blockers (catheter with inflatable balloon attached to the tip) have been described and are used in routine clinical practice [3]. Both airway devices have been manufactured in smaller sizes to accommodate children, but due to the small size of pediatric airways, the smallest double-lumen endotracheal tubes can usually only be used in patients 8 years of age and older. This guideline is derived from pediatric patients that fall within a spectrum of normal physical development. In our case, the patient was 11 years old but diagnosed with growth delay and short stature: her height and weight were $1.18 \mathrm{~m}$ and $24 \mathrm{~kg}$, respectively, corresponding to the 0.1 percentile for height and 0.43 percentile for weight. Based on this, we were concerned that a standard 26 French (Fr) double-lumen tube may be too large to be accommodated by the patient's airway. The use of a bronchial blocker was in theory possible, but while it would make a conventional surgery feasible (for example any surgical resection of the non-ventilated lung), it did not support a lung lavage for the following reason: during a lung lavage, one lung needs to be continuously ventilated, while the other lung requires lung isolation with a device that provides an access port through which lavage solution can be instilled and withdrawn (a double-lumen endotracheal tube). A bronchial blocker provides lung isolation but does not have an access port.

The complexities of this patient's diagnosis warranted an alternative evaluation to ensure the procedure could be performed safely and to minimize patient discomfort. Simulating the procedure in a safe environment had the following advantages: a) reduction of care team member stress on the day of the procedure; $b$ ) reduction of the risk of damaging the airway; and c) reduction of the risk of cancelling the procedure due to the inability to secure the airway. The purpose of this work was to confirm if a $26 \mathrm{Fr}$ double-lumen endotracheal tube could be used to successfully intubate this pediatric patient.

\section{Methods}

Procedural planning processes incorporate medical knowledge, a patient diagnosis, and images of the patient's anatomy to formulate a medical protocol. For many medical procedures this is a routine process. Rarer cases with their increased complexities raise the uncertainty of procedural outcomes. The objective for this work was to develop an efficient methodology that incorporates new technologies to reduce the procedural uncertainties surrounding patient's anatomical size and the fit of a medical device. The new methodology was applied to a case where the medical team needed to confirm the fit of a medical device for a challenging pediatric patient.

\section{Constraints}

Selection of an airway device by conventional methods was, in our reported case, constrained by the patient and the procedure. If an operation requires one-lung isolation in an adult patient, multiple methods to secure the airway are available to the anesthesiologist. For pediatric patients, the size of the airway presents limitations and precludes the use of some airway devices. The procedure presented in this manuscript introduced further constraints as some airway devices that could typically be used in a pediatric patient would make the conduct of a lung lavage impossible.

The current standard to evaluate internal anatomical size is by having a radiologist read the images from a patient's scan. The images are captured perpendicular to the patient's body to show a cross-section of the anatomy. The trachea does not follow a plumb line from the mouth to the feet, but rather slopes backwards toward the spine. Therefore, capturing measurements through this method is inherently inaccurate because the anatomy runs at an oblique angle to the twodimension (2D) images. Due to the size of the patient for the procedure presented here, the team could not confidently select a procedural method based off the radiologist reading.

\section{Evaluation metrics}

Traditional elements of procedural success are efficiency of the procedure and patient outcome. For the purposes of this evaluation we also considered the cost. At the time of this publication, the developed techniques are not reimbursable by insurance and therefore the cost of these services must be supported by research funds or passed along to the patient. The cost of these techniques is a critical driver towards hospital adoption and a barrier to entry; therefore, a hospital must see a return on their investment. The opportunity to reduce operating room time, reduce patient recovery time, or improve surgeon confidence can significantly impact the cost of a procedure and are important reasons to include new technologies into the procedural planning process.

\section{Results}

The results of this work include three components: 1) a process that can be replicated to evaluate the fit of any medical device in a patient, 2) a clinical review of the models to 
confirm the method, and 3) a financial review to support the feasibility of the method.

\section{Process}

The first result of this work is a process that can be used to evaluate the fit of a medical device within a specific patient's anatomy. The following description is described within the context of our use case, as described previously. Anatomical models of the trachea, bronchi, and lungs were created from computed tomography (CT) images. The double lumen endotracheal tube was reverse engineered to develop a computer aided design (CAD) model of the device. Two feasibility studies were prepared to confirm the fit of the device within the anatomy.

Anatomical model Computed tomography images were captured of the patient's anatomy to create a model of the airways. A Siemens CT scanner captured the patient's chest without contrast at a scan resolution of $0.5 \mathrm{~mm} \times 0.5 \mathrm{~mm} \times 3.0 \mathrm{~mm}$. The scans were segmented using Mimics (Materialise NV, Leuven, Belgium). A threshold of -1024 Hounsfield unit (HU) to $-500 \mathrm{HU}$ was applied to isolate the air volume within the trachea and bronchi. The air volume was wrapped at two thicknesses $(0.5 \mathrm{~mm}$ and $1.5 \mathrm{~mm})$ in 3-matic (Materialise NV, Leuven, Belgium) to create the walls of the trachea and bronchi walls. A Boolean subtraction removed the air volume from trachea and bronchi wall models. The ends of the models were cropped to access the air volume within the anatomical region. Models of the lungs and rib cage were segmented as solid models from the CT images to serve as a reference point during the procedure planning process.

Medical device model A 26 Fr left-sided double lumen endotracheal tube was obtained from Teleflex Incorporated (Research Triangle Park, NC, USA). The double lumen endotracheal tube was reversed engineered to produce CAD models in SOLIDWORKS (Dassault Systèmes, VélizyVillacoublay, France). The blue and white cuffs (balloons) on the device were modeled separately from the tube of the double lumen endotracheal device. The visibility of the blue and white cuffs will be toggled in the virtual environment to evaluate the size differential between the tube, cuffs, and anatomy.

Virtual reality environment The $0.5 \mathrm{~mm}$ thick trachea and double lumen endotracheal tube models were aligned and colored in Meshlab (ISTI-CNR, Pisa, Italy) and exported to standard 3D object (OBJ) files. The models were loaded into the virtual reality based Interactive Multi-touch Table $[4,5]$ to display the models. The head-tracked stereoscopic glasses aligned the model to the visual perspective of the primary

user. The system was used to evaluate the feasibility of inserting the double lumen trachea tube into the anatomy.

Three-dimensional printing The $1.5 \mathrm{~mm}$ thick trachea and bronchi model were printed with a Stratasys (Eden Prairie, MN) J750 three-dimension (3D) printer. The model was printed with a material combination of VeroPureWhite, AgilusClear, and VeroClear to create a pliable transparent model to evaluate the fit of endotracheal tube inside the anatomy (See Table 1).

\section{Clinical review}

The second result is a clinical evaluation and procedural confirmation for the effectiveness of the process in addition to conventional procedural planning methods. The patient's airway model and double-lumen trachea medical device model were evaluated twice in preparation for the procedure. The first evaluation used the virtual replica of the patient's anatomy, while the second evaluation used a physical replica.

Evaluation The attending pediatric anesthesiologist for this case met with the medical device team and walked-through the airway in a $3 \mathrm{D}$ virtual environment. The $3 \mathrm{D}$ virtual environment allowed free manipulation of the rendered doublelumen endotracheal tube and patient's anatomy.

First, the patient's airways were measured. The 3D virtual model was necessary to rapidly identify cross-section planes perpendicular to the patient's airway. The cross-sectional planes were used to measure the anatomy at key locations. The narrowest diameter of the airway measured $9.1 \mathrm{~mm}$ at the trachea level and $8.1 \mathrm{~mm}$ at the left main bronchus level (Fig. 1). The virtual measurements were compared to the 2D in-plane measurements captured by the team. The comparison confirmed that the anatomy was rendered at the same scale as the patient and also confirmed the inaccuracy of the measurements captured by the radiologist. The preliminary measurements were compared to the measured endotracheal tube outer diameter $(8.5 \mathrm{~mm}$ at site that corresponds to placement in trachea, $7.5 \mathrm{~mm}$ at site that corresponds to placement in left main bronchus) were encouraging (Table 2).

Second, the endotracheal tube model was virtually introduced into the trachea and bronchi passageways to confirm the fit of the device (Fig. 2). During this fitting, the visibility of

Table 1 Material used to make a 3D printed model

\begin{tabular}{ll}
\hline Material & Material Amount (g) \\
\hline VeroPureWhite & 12 \\
AgilusClear & 25 \\
VeroClear & 17 \\
Support Amount & 61 \\
\hline
\end{tabular}


Fig. 1 Double lumen endotracheal tube (left) and computer-aided design (CAD) model (right). The white and blue cuffs can be expanded to isolate each lung

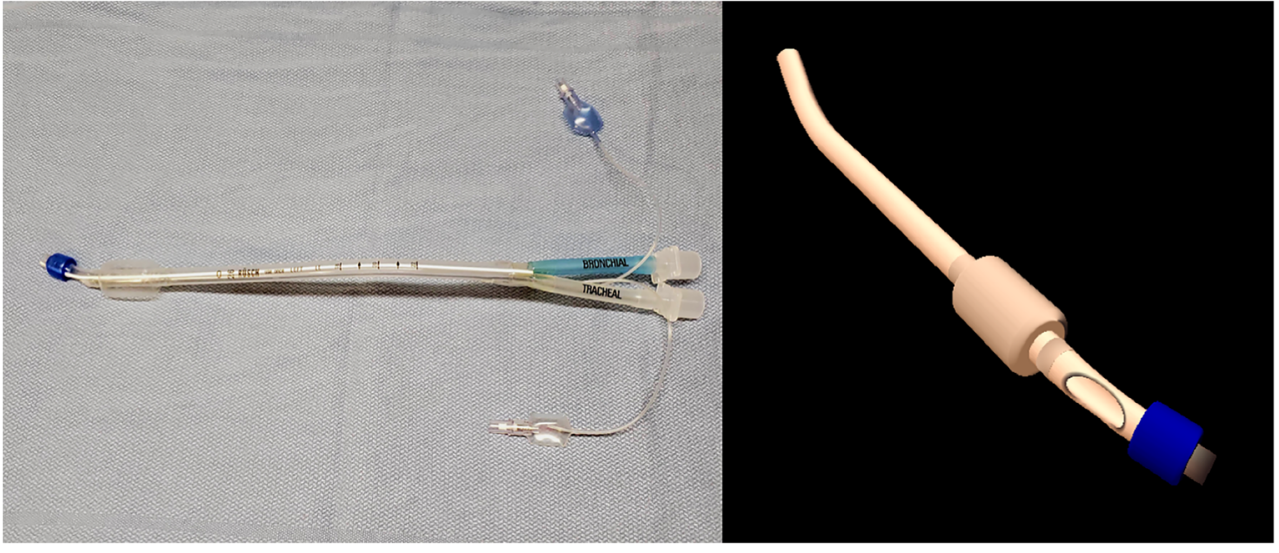

the blue and white cuff models was toggled to compare the tube and cuff diameters with the surface of the anatomy. The virtual fit evaluation confirmed the double-lumen trachea tube device would isolate the lung for the procedure.

Finally, the 3D model of the airway was printed which allowed the simulated introduction of the real double-lumen endotracheal tube (Fig. 3). In this last check-point, the airway size also proved to be large enough to accommodate the $26 \mathrm{Fr}$ double-lumen endotracheal tube.

Based on the virtual and physical evaluations, it was concluded that the use of the $26 \mathrm{Fr}$ double lumen tube in this particular patient was likely feasible and proceeded with the planned sequential whole-lung lavage (Fig. 4).

Confirmation On the day of the procedure, a peripheral intravenous catheter was placed. The patient received midazolam premedication and was induced with propofol and fentanyl. After successful mask ventilation was established the patient was given a neuromuscular relaxant (rocuronium). With the help of a C-MAC videolaryngoscope (Karl Storz Endoscopy, El Segundo, CA), a Cormack-Lehane grade 1 view was obtained, and the 26 Fr double-lumen endotracheal tube was placed according to manufacturer's recommendations. Passage of the lubricated tube was noted to be smooth without resistance. Correct placement was confirmed by fiberoptic bronchoscopy (Olympus Exera BF-XP160, Olympus

Table 2 Measurements of patient's anatomy and the double lumen trachea tube medical device to confirm the fit

\begin{tabular}{lll}
\hline Measurements & Trachea & Left Bronchi \\
\hline 2D plane measurements & $9.8 \mathrm{~mm}$ & $7.6 \mathrm{~mm}$ \\
Virtual measurements of patient's anatomy & $9.1 \mathrm{~mm}$ & $8.1 \mathrm{~mm}$ \\
Diameter of double lumen trachea tube & $8.5 \mathrm{~mm}$ & $7.5 \mathrm{~mm}$ \\
Expanded diameter of white cuff & $19.8 \mathrm{~mm}$ & $*$ N/A \\
Expanded diameter of blue cuff & $* \mathrm{~N} / \mathrm{A}$ & $14.2 \mathrm{~mm}$ \\
\hline
\end{tabular}

*N/A = not applicable
America Medical, Center Valley, PA). The blue and white cuff were inflated according to manufacturer's recommendations and the patient was ventilated with the following settings: pressure-control, positive inspiratory pressure $24 \mathrm{~cm}$ $\mathrm{H}_{2} \mathrm{O}$, respiratory rate $18 / \mathrm{min}$, positive end-expiratory pressure $5 \mathrm{~cm} \mathrm{H}_{2} \mathrm{O}$, inspired oxygen concentration $100 \%$. A second peripheral intravenous catheter and an arterial catheter for cardiorespiratory monitoring were placed and the procedure was started. Left lung isolation was successfully achieved, and the ventilator was adjusted to account for single-lung ventilation. A total of $5 \mathrm{~L}$ warmed $0.9 \%$ saline solution was instilled in aliquots and consecutively removed. The patient tolerated the procedure well. At the conclusion of the procedure, doublelung ventilation was resumed, and the patient was brought to the intensive care unit where she was extubated on postoperative day \#1. The patient underwent a successful right-sided lung lavage 7 days later. The same lung isolation method was used.

\section{Financial cost}

The third result was the cost to complete the virtual and physical evaluations. Healthcare costs across the United States continue to rise and healthcare providers continually evaluate the financial incentives of new methods. A financial analysis provides the evidence to support the feasibility of this method.

The Bakken Medical Devices Center (BMDC) at the University of Minnesota supported this case. Their technical expertise includes anatomical segmentation, reverse engineering, virtual reality, and three-dimensional printing. The BMDC is an at-cost service center. The complexity of the task determines if a graduate student $(\$ 56.75$ per hour) or undergraduate student (\$18.91 per hour) completes the project. Machine time includes either software costs or physical machinery needed to complete the project. The final cost to complete the process for this procedure was $\$ 1522.26$ (See Table 3). The costs are dependent on the size and complexity of the patient anatomy modeled. 

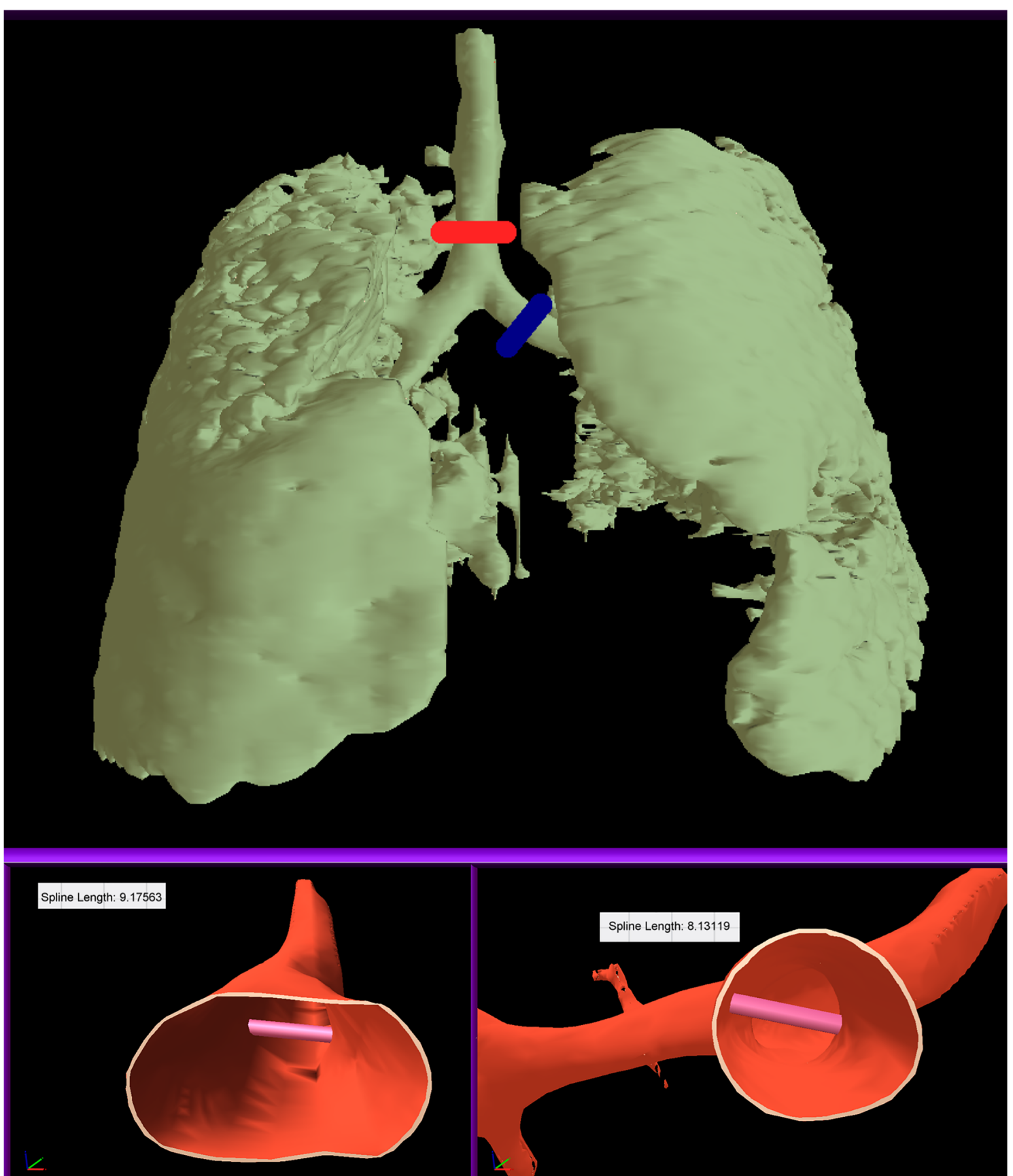

Fig. 2 Virtual reality models of the trachea and lungs to compare multiple inner diameters of the trachea with the outer diameters of the double lumen endotracheal tube

\section{Discussion}

Technology in medicine has for a long time maintained an established role in diagnosis and treatment of diseases. In recent years, progress in this field has accelerated significantly, leading to new unique applications. The recent surgical literature features frequent examples of technology including digital design, 3D modeling, and 3D printing that are being used for preoperative procedure planning and trainee education [6-9].
Anesthesiology is a field that has been a leader and traditionally been advancing patient safety with the help of technological inventions. Examples include, but are not limited to: the introduction of pulse oximetry [10] and capnography [11] to detect respiratory problems; the use of transesophageal echocardiography [12] to rapidly assess cardiac function and aid the cardiothoracic surgeon in evaluation of a repaired heart valve; and the implementation of advanced monitoring systems that utilize arterial waveform analysis within goaldirected therapy protocols to improve patient outcomes in 
Fig. 3 Virtual fit evaluation with the double lumen endotracheal tube placed inside of the trachea model
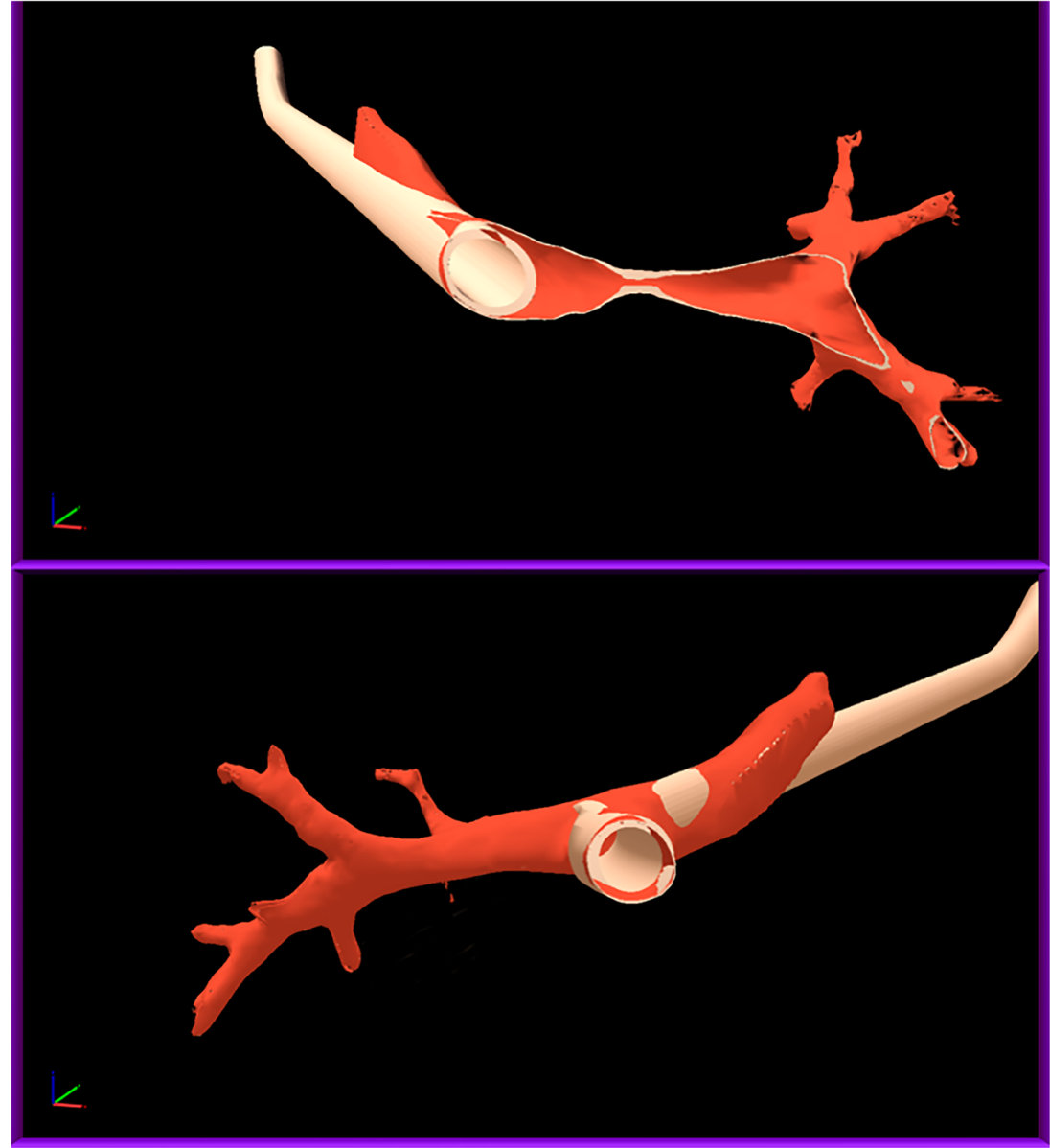

the perioperative period [13]. More recently, near infra-red spectroscopy is gaining increasing confidence in ensuring adequate oxygen delivery to the brain and kidney and, the bispectral index is being utilized to titrate sedative effects of anesthetics.

The specialty of anesthesiology has so far been less visible in the area of 3D-technology, but interest is increasing as shown by recent publications. Pedersen et al. [14] developed a 3D-printed bronchial tree simulator and compared it with commercially available simulators for tasks including localization of right upper lobe bronchial lumen, bronchial blocker placement and fluid aspiration. Study participants rated the 3D-printed model overall significantly more realistic compared with the two commercially available simulators. In addition, the cost for the 3D-printed model was significantly lower. Chao et al. [15] published a systematic review of the application of three-dimensional printing technology in anesthesia. Their comprehensive analysis included the timeframe of January 1990 to June 2016. Thirty-four articles met inclusion criteria, 8 of which were related to the field of anesthesia. In those 8 articles, authors described the use of 3D printing for pre-procedure planning, preparation, education, and training. Wilson et al. [16] were faced with a similar situation of a pediatric patient requiring lung isolation for whole lung lavage. The group also utilized a $3 \mathrm{D}$-print model to practice various methods of lung isolation prior to the procedure. Given the age of the patient (6 years), a double-lumen endotracheal tube could not be used, and lung isolation was achieved by placement of two separate single-lumen endotracheal tubes and subsequent advancement of one single-lumen endotracheal tube into the left mainstem bronchus. While our patient underwent a similar procedure, this manuscript adds the novel use of digital three-dimensional modeling and the use of a virtual reality environment to assess lung isolation methods.

The rationale for using virtual reality and $3 \mathrm{D}$ printing for a single patient was to validate the use of each emerging technology. Virtual reality and 3D printing are complementary technologies that can be leveraged in unique ways for medical use cases. In this use case, the first advantage of using a virtual reality model was to capture measurements of the anatomy and compare them with the medical device. The second advantage was the ability to introduce the medical device into the anatomy and analyze the cross-sections at key locations. The cross-sectional views captured perpendicular to the airway showed if the medical device overextended into the tissue 
Fig. 4 Fit evaluation using a 3Dprinted model of the patient's trachea and the proposed $26 \mathrm{Fr}$ double lumen endotracheal tube from the manufacturer

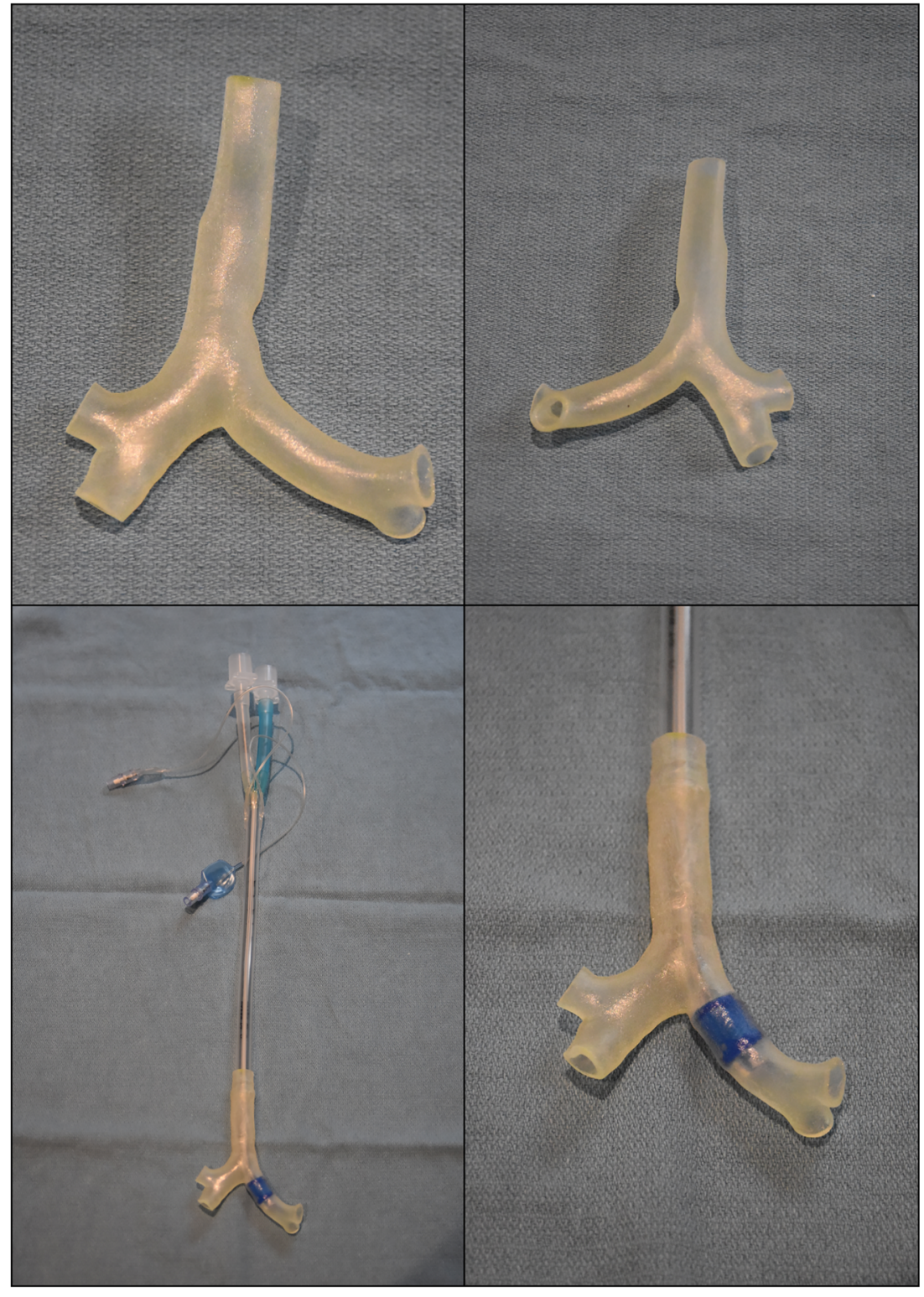

Table 3 The costs for labor, materials, and machine use for the virtual and physical trachea and bronchi model

\begin{tabular}{lllll}
\hline Service & Item & Time $(\mathrm{hr})$ & Cost $(\$)$ & Total $(\$)$ \\
\hline \multirow{2}{*}{ Segmentation } & Labor & 7 & $\$ 56.75$ & $\$ 397.25$ \\
& Machine time & 7 & $\$ 15.00$ & $\$ 105.00$ \\
CAD & Labor & 20 & $\$ 18.91$ & $\$ 378.20$ \\
& Machine time & 20 & $\$ 15.00$ & $\$ 300.00$ \\
Visualization & Labor & 3 & $\$ 56.74$ & $\$ 170.22$ \\
& Machine time & 3 & $\$ 15.00$ & $\$ 45.00$ \\
3D Printing & Materials & 1 & $\$ 15.24$ & $\$ 15.24$ \\
& Labor & 1 & $\$ 18.91$ & $\$ 18.91$ \\
& Machine Time & 3.5 & $\$ 26.41$ & $\$ 92.44$ \\
& & & & $\$ 1522.26$ \\
\hline
\end{tabular}

which could potentially have caused patient discomfort and airway injuries. Furthermore, prior to the procedure, the attending anesthesiologist was able to freely navigate the bronchial tree in a $3 \mathrm{D}$ environment which provided the opportunity to become familiar with the anatomy and to recognize potential problem areas.

The 3D printed model provided additional benefits not available with the virtual reality model. The primary advantage was the ability to simulate the procedure and experience the tactile response when introducing the medical device. The tactile knowledge was transferred to the operating room for real-time feedback that the procedure was going as planned. We found the use of both technologies was important to increase procedural confidence. We envision a future where virtual reality and $3 \mathrm{D}$ printing technologies are standard to plan every procedure. 
Our case illustrates the possibilities of preoperative planning by using computer simulation and true-scale models obtained from patient imaging. The ability to evaluate feasibility of airway management techniques in the safety of a simulated environment greatly adds to overall patient safety. With respect to our case in the field of anesthesiology, securement of the airway is a critical step in patient management and, similar to the take-off in the commercial airline industry, represents a time period in which errors or unanticipated problems can rapidly lead to morbidity and even mortality. The scheduled procedure (lung lavage) required lung isolation for which options are quite limited in the pediatric population. Adding to the complexity of the situation was the fact that our patient's height and weight, which directly relates to airway anatomy and size, were small compared to children of similar age. The ability to use our airway device of choice (double-lumen endotracheal tube) in a virtual airway environment and in a 3D-printed airway model gave us assurance that its use would be feasible in this patient. As the use of virtual reality technology grows, we anticipate increased access to medical device CAD models which will further reduce the cost of this method. If we had discovered that a fit was likely not achievable, alternative routes of airway securement could have been evaluated in the safe simulation environment, thereby avoiding a stressful situation on the day of the procedure, potential damage to the airway and cancellation of the procedure due to inability to provide the necessary airway isolation.

Our case study has several limitations. As described earlier, the costs for the combined creation of an anatomical model, a device model, a virtual reality environment and a 3D printed airway model are currently high. Since this case was a pilot feasibility evaluation, we decided to use all available technologies to gain additional experience with the overall process and to assess the value of each component. As further investigations into this area are conducted, clinicians and investigators may re-define the need for the different components. In addition, costs will likely decrease as mentioned in the previous paragraph. Another limitation is related to airway tissue pliability: cartilage rings found in the trachea and bronchi are rigid, but the posterior part of the airway is formed by a flexible membranous wall that results in expansion when subjected to a distending force. At this point, tissue flexibility is difficult to replicate in printed 3D models which should be kept in mind when assessing the medical device fit.

In conclusion, our manuscript describes a successful process to use 3D-modeling and 3D-printing technologies to confirm the fit of a medical device within a patient's specific anatomy prior to the procedure. With further advancements in technology and reductions in material and production costs, an appealing future prospect is the individualized evaluation of patients with difficult anatomies that may include computer modeling, 3D-simulations and 3D-models that can be used to study different airway securement approaches without endangering the patient's life. The high fidelity and realism is nicely depicted in a letter to the editor by Bustamante et al. [17] where the authors show fiberoptic bronchoscopy pictures from a patient's airway compared to pictures from a 3Dprinted model.

Authors' contributions Name: Benjamin Kloesel, MD, MSBS.

Contribution: This author helped conceptualize the project, write, edit, and revise the manuscript.

Name: Bethany Juhnke, M.S.

Contribution: This author helped conceptualize the project, write, edit, and revise the manuscript.

Name: Laura Irvine.

Contribution: This author helped edit and revise the manuscript.

Name: James V Donadio IV.

Contribution: This author helped edit and revise the manuscript.

Name: Arthur Erdman, Ph.D.

Contribution: This author helped edit and revise the manuscript.

Name: Kumar Belani, MBBS, MS, FAAP.

Contribution: This author helped conceptualize the project, edit and revise the manuscript.

Funding Funding was provided by departmental funds.

\section{Compliance with ethical standards}

Conflict of interest All involved authors declare no conflicts of interest.

Consent to participate and consent for publication The patient's family (legal guardian) provided written consent for publication.

\section{References}

1. Griese, M., Pulmonary alveolar proteinosis: a comprehensive clinical perspective. Pediatrics. 140:(2), 2017. https://doi.org/10.1542/ peds.2017-0610

2. Awab, A., Khan, M. S., Youness, H. A., Whole lung lavagetechnical details, challenges and management of complications. J. Thorac. Dis. 9 (6):1697-1706, 2017. https://doi.org/10.21037/jtd. 2017.04.10

3. Falzon, D., Alston, R. P., Coley, E., Montgomery, K., Lung Isolation for Thoracic Surgery: From Inception to EvidenceBased. J. Cardiothorac. Vasc. Anesth. 31 (2):678-693, 2017. https://doi.org/10.1053/j.jvca.2016.05.032

4. Coffey, D., Malbraaten, N., Bao, T. B., Borazjani, I., Sotiropoulos, F., Erdman, A. G., Keefe, D. F., Interactive slice WIM: navigating and interrogating volume data sets using a multisurface, multitouch VR interface. IEEE Trans. Vis. Comput. Graph. 18(10):1614-1626. 2012. https://doi.org/10.1109/TVCG.2011.283

5. Coffey, D., Malbraaten, N., Le, T., Borazjani, I., Sotiropoulos, F., Keefe, D.F., Slice WIM: a multisurface, multitouch interface for overview+detail exploration of volume datasets in virtual reality. Paper presented at the Symposium on Interactive 3D Graphics and Games, San Francisco, CA, 2011.

6. Andolfi, C., Plana, A., Kania, P., Banerjee, P. P., Small, S., Usefulness of Three-Dimensional Modeling in Surgical Planning, Resident Training, and Patient Education. J. Laparoendosc. Adv. Surg. Tech. A 27(5):512-515. 2017. https://doi.org/10.1089/lap. 2016.0421

7. Chen, S. A., Ong, C. S., Malguria, N., Vricella, L. A., Garcia, J. R., Hibino, N., Digital Design and 3D Printing of Aortic Arch 
Reconstruction in HLHS for Surgical Simulation and Training. World J. Pediatr. Congenit. Heart. Surg. 9(4):454-458, 2018. https://doi.org/10.1177/2150135118771323

8. Ganguli, A., Pagan-Diaz, G. J., Grant, L., Cvetkovic, C., Bramlet, M., Vozenilek, J., Kesavadas, T., Bashir, R., 3D printing for preoperative planning and surgical training: a review. Biomed. Microdevices 20(3):65, 2018.https://doi.org/10.1007/s10544-0180301-9

9. Tetsworth, K., Block, S., Glatt, V., Putting 3D modelling and 3D printing into practice: virtual surgery and preoperative planning to reconstruct complex post-traumatic skeletal deformities and defects. SICOT J 3:16, 2017. https://doi.org/10.1051/sicotj/2016043

10. Van Meter, A., Williams, U., Zavala, A., Kee, J., Rebello, E., Tsai, J., Ifeanyi, I., Ruiz, J., Lim, J., Owusu-Agyemang, P., Beat to Beat: A Measured Look at the History of Pulse Oximetry. J. Anesth. Hist. 3(1):24-26, 2017. https://doi.org/10.1016/j.janh.2016.12.003

11. Cook, T. M., The winds of change-progress in the implementation of universal capnography. Anaesthesia 71(4):363-368, 2016. https://doi.org/10.1111/anae.13387

12. Vegas, A., Meineri, M., Core review: three-dimensional transesophageal echocardiography is a major advance for intraoperative clinical management of patients undergoing cardiac surgery: a core review. Anesth. Analg. 110(6):1548-1573, 2010. https://doi.org/10. 1213/ANE.0b013e3181d41be7
13. Mehta, N., Fernandez-Bustamante, A., Seres, T., A review of intraoperative goal-directed therapy using arterial waveform analysis for assessment of cardiac output. Sci. World J. 2014:702964, 2014. https://doi.org/10.1155/2014/702964

14. Pedersen, T. H., Gysin, J., Wegmann, A., Osswald, M., Ott, S. R., Theiler, L., Greif, R., A randomised, controlled trial evaluating a low cost, 3D-printed bronchoscopy simulator. Anaesthesia 72 (8): 1005-1009, 2017. https://doi.org/10.1111/anae.13951

15. Chao, I., Young, J., Coles-Black, J., Chuen, J., Weinberg, L., Rachbuch, C., The application of three-dimensional printing technology in anaesthesia: a systematic review. Anaesthesia 72 (5):641650, 2017. https://doi.org/10.1111/anae.13812

16. Wilson, C. A., Arthurs, O. J., Black, A.E., Schievano, S., Hunt, C., van Hoog, S., Wallis, C., Sury, M. R., Printed three-dimensional airway model assists planning of single-lung ventilation in a small child. Br. J. Anaesth. 115(4):616-620, 2015. https://doi.org/10. 1093/bja/aev305

17. Bustamante, S., Shravan Cheruku, M. D., 3D Printing for Simulation in Thoracic Anesthesia. J. Cardiothorac. Vasc. Anesth. 30(6):e61-e63, 2016. https://doi.org/10.1053/j.jvca.2016. 05.044

Publisher's Note Springer Nature remains neutral with regard to jurisdictional claims in published maps and institutional affiliations. 